\title{
Stabilization of nonlinear systems using state-feedback periodic event-triggered controllers
}

\author{
W. Wang, R. Postoyan, D. Nešić and W.P.M.H. Heemels
}

\begin{abstract}
We investigate the scenario where a controller communicates with a plant at discrete time instants generated by an event-triggering mechanism. In particular, the latter collects sampled data from the plant and the controller at each sampling instant, and then decides whether the control input needs to be updated, leading to periodic event-triggered control (PETC). In this paper, we propose a systematic design procedure for PETC that stabilize general nonlinear systems. The design is based on the existence of a continuous-time statefeedback controller, which stabilizes the system in the absence of communication constraints. We then take into account the sampling and we design an event-triggering condition, which is only updated at some of the sampling instants, to preserve stability. An explicit bound on the maximum sampling period with which the triggering rule is evaluated is provided. We show that there exists a trade-off between the latter and a parameter used to define the triggering condition. The results are applied to a van de Pol oscillator as an illustration.
\end{abstract}

\section{INTRODUCTION}

Major advancements over the last decades in wired and wireless communication networks gave rise to networked control systems (NCS). These are systems in which the sensors and the actuators communicate with the controller via a shared digital channel. A major challenge in this context is to design control strategies which do not "overuse" the network, to limit the transmission delays and the occurence of packet losses, which may destroy the desired closedloop system properties. An attractive solution consists in adapting the transmissions to the current state of the plant, we talk of event-triggered control, see [5] and the references therein. This paradigm consists in continuously evaluating a state/output-dependent condition and, when the latter is satisfied, a transmission is triggered. Many works have shown that event-triggered control is able to significantly reduce the number of transmissions compared to the traditional periodic sampling, see [9], [10], [13], [21] for instance. Nevertheless, the continuous evaluation of the triggering condition is

This work is supported by the Australian Research Council under the Discovery Project DP1094326, the ANR under the grant COMPACS (ANR13- BS03-0004-02), and the Innovational Research Incentives Scheme under the VICI grant Wireless control systems: A new frontier in automation (No. 11382) awarded by NWO (The Netherlands Organisation for Scientific Research) and STW (Dutch Technology Foundation).

W. Wang and D. Nešić are with the Department of Electrical and Electronic Engineering, The University of Melbourne, Parkville, 3010, Victoria, Australia wweiqustegmail.com, dnesiceunimelb.edu.au

R. Postoyan is with the Université de Lorraine, CRAN, UMR 7039 and the CNRS, CRAN, UMR 7039, France romain.postoyaneuniv-lorraine.fr

W.P.M.H. Heemels is with the Department of Mechanical Engineering, Eindhoven University of Technology, Eindhoven, The Netherlands, m. heemels@tue.nl not possible when the implementation platform is digital. Instead, the triggering criterion can only be evaluated at some sampling instants, leading to the periodic event-triggered control (PETC), see [3], [4], [6] and the references therein.

Results on the design of PETC for linear systems are presented, for instance, in [3], [4]. In [6], a methodology is proposed for nonlinear systems. The idea is to start from a given event-triggered controller and to redesign it to obtain a periodic event-triggered controller, based on a condition on the successive Lie derivatives of the original triggering condition. A bound on the sampling period is provided, which is based on the minimum inter-transmission time of the event-triggered controller, which is often difficult to precisely estimate. On the other hand, the generic results in [18] on the sampling of hybrid controllers show that, if an eventtriggered controller ensures a uniform global asymptotic stability property, the latter is preserved semiglobally and practically when emulating the controller with sufficiently fast sampling.

In this paper, we design periodic event-triggered controllers for nonlinear systems using a different approach compared to [6], [18]. We start from a continuous-time controller which stabilizes the plant in the absence of network (and not an event-triggered controller as in [6], [18]). We then take into account the communication network and we design a triggering condition, which is only evaluated at given sampling instants. The stability of the overall system is guaranteed provided that the maximum sampling period, with which the triggering rule is evaluated, is less than a given bound. We model for that purpose the overall system as a hybrid system using the formalism of [2] and the analysis relies on the construction of a novel hybrid Lyapunov function. The results are applied to a van de Pol oscillator as an illustration.

Compared to [6], the bound on the sampling period does not rely on the estimation of the minimum inter-transmission time of a predesigned event-triggered controller, and is therefore easier to compute. Furthermore, the triggering condition we propose is easy to construct as we only need to verify an input-to-state stability property, as opposed to a local condition on the Lie derivatives of the triggering rule of the original event-triggered controller in [6]. In addition, our results clearly show that there is a trade-off between the parameter of the triggering condition and the maximum time between two sampling instants. Also, the sampling instants at which the triggering rule is evaluated are not necessarily periodic. On the other hand, we cope with a more specific type of triggering rules than in [6]. In contrast with [18], 
we provide an explicit bound on the sampling period and we ensure uniform global asymptotic stability properties, as opposed to uniform semiglobal practical stability.

The paper is organized as follows. The notation and preliminaries on hybrid systems are given in Section II. We state the problem and present the model in Section III. The main results are stated in Section IV. Simulation results and conclusions are respectively provided in Sections V and VI. The proofs are given in the appendix.

\section{Preliminaries}

Let $\mathbb{Z}_{>0}:=\{1,2, \cdots\}, \mathbb{Z}_{\geq 0}:=\{0,1,2, \cdots\}$ and $\mathbb{R}_{\geq 0}:=$ $[0, \infty)$. Let $|x|$ denote the Euclidean norm of the vector $x \in \mathbb{R}^{n}$. For $(x, y) \in \mathbb{R}^{n+m},(x, y)$ stands for $\left[x^{T}, y^{T}\right]^{T}$. Given a set $\mathcal{A} \subset \mathbb{R}^{n}$ and $x \in \mathbb{R}^{n}$, we define the distance of $x$ to $\mathcal{A}$ as $|x|_{\mathcal{A}}:=\inf _{y \in \mathcal{A}}|x-y|$. A set-valued mapping $M: \mathbb{R}^{m} \rightrightarrows \mathbb{R}^{n}$ is locally bounded if, for any $x \in \mathbb{R}^{m}$, there exists a neighborhood $U_{x}$ of $x$ such that $M\left(U_{x}\right)$ is a bounded set. A set-valued mapping $M: \mathbb{R}^{m} \rightrightarrows \mathbb{R}^{n}$ is outer semi-continuous when its graph $\left\{(y, z) \in \mathbb{R}^{m+n}\right.$ : $z \in M(y)\}$ is closed, see Lemma 5.10 in [2]. A function $\gamma: \mathbb{R}_{\geq 0} \rightarrow \mathbb{R}_{\geq 0}$ is of class- $\mathcal{K}$, if it is continuous, zero at zero and strictly increasing and it is of class- $\mathcal{K}_{\infty}$ if, in addition, it is unbounded. A function $\gamma: \mathbb{R}_{\geq 0}^{2} \rightarrow \mathbb{R}_{\geq 0}$ is of class- $\mathcal{K} \mathcal{L}$, if it is continuous, for each $r \in \mathbb{R}_{\geq 0}, \gamma(\cdot, r)$ is of class- $\mathcal{K}$, and, for each $s \in \mathbb{R}_{\geq 0}, \gamma(s, \cdot)$ is decreasing to zero. For $x, v \in \mathbb{R}^{n}$ and locally Lipschitz $U: \mathbb{R}^{n} \rightarrow \mathbb{R}$, let $U^{\circ}(x ; v)$ be the Clarke derivative of the function $U$ at $x$ in the direction $v$, i.e. $U^{\circ}(x ; v):=\limsup _{y \rightarrow x, \lambda \downarrow 0} \frac{U(y+\lambda v)-U(y)}{\lambda}$. This notion will be useful as we will be working with locally Lipschitz Lyapunov functions, which are not differentiable everywhere.

Consider the following hybrid system [2]

$$
\begin{array}{rrr}
\dot{q}=\mathcal{F}(q) & & q \in C \\
q^{+} \in \mathcal{G}(q) & & q \in D
\end{array}
$$

with state $q \in \mathbb{R}^{n}$ and where $C, D \subset \mathbb{R}^{n}$ are respectively the flow and the jump sets. We assume that: the sets $C$ and $D$ are closed; $\mathcal{F}: \mathbb{R}^{n} \rightarrow \mathbb{R}^{n}$ is a continuous function; $\mathcal{G}$ : $\mathbb{R}^{n} \rightrightarrows \mathbb{R}^{n}$ is outer semi-continuous and locally bounded; and $\mathcal{G}(q)$ is nonempty for each $q \in D$.

We now recall some definitions from [2]. A set $S \subset$ $\mathbb{R}_{>0} \times \mathbb{Z}_{>0}$ is called a compact hybrid time domain if $S=\bigcup_{j=0}^{J-1}\left(\left[t_{j}, t_{j+1}\right], j\right)$ for some finite sequence of times $0=t_{0} \leq t_{1} \leq t_{2} \leq \cdots \leq t_{J}$. The set $S$ is a hybrid time domain if for all $(T, J) \in S, S \cap([0, T] \times\{0,1, \cdots, J\})$ is a compact hybrid time domain. A function $q: S \rightarrow \mathbb{R}^{n}$ is a hybrid arc if $S$ is a hybrid time domain and $q(\cdot, j)$ is locally absolutely continuous for each $j$. A hybrid arc $q: \operatorname{dom} q \rightarrow \mathbb{R}^{n}$ is a solution to (1) if $q(0,0) \in C \cup D$ and

1) for all $j \in \mathbb{Z}_{\geq 0}$ and almost all $t$ such that $(t, j) \in \operatorname{dom} q$, $q(t, j) \in C$ and $\dot{q}(t, j)=\mathcal{F}(q(t, j))$;

2) for all $(t, j) \in \operatorname{dom} q$ such that $(t, j+1) \in \operatorname{dom} q$, $q(t, j) \in D$ and $q(t, j+1) \in \mathcal{G}(q(t, j))$.

A solution is maximal if it cannot be extended and it is complete when $\operatorname{dom} q$ is unbounded. We also recall the following set stability definition.
Definition 1: The closed set $\mathcal{A} \subset \mathbb{R}^{n}$ is called uniformly globally asymptotically stable (UGAS) for system (1) if there exists $\beta \in \mathcal{K} \mathcal{L}$ such that all solutions $q$ to system (1) satisfy

$$
|q(t, j)|_{\mathcal{A}} \leq \beta\left(|q(0,0)|_{\mathcal{A}}, t+j\right) \quad \forall(t, j) \in \operatorname{dom} q
$$

and all maximal solutions to system (1) are complete.

We will need the following result, which corresponds to Lemma II.1 in [8].

Lemma 1: Consider two functions $U_{1}: \mathbb{R}^{n} \rightarrow \mathbb{R}$ and $U_{2}: \mathbb{R}^{n} \rightarrow \mathbb{R}$ that have well-defined Clarke derivatives for all $x \in \mathbb{R}^{n}$ and $v \in \mathbb{R}^{n}$. Introduce three sets $A:=$ $\left\{x: U_{1}(x)>U_{2}(x)\right\}, B:=\left\{x: U_{1}(x)<U_{2}(x)\right\}, \Gamma:=$ $\left\{x: U_{1}(x)=U_{2}(x)\right\}$. Then, for any $v \in \mathbb{R}^{n}$, the function $U(x):=\max \left\{U_{1}(x), U_{2}(x)\right\}$ satisfies $U^{\circ}(x ; v)=U_{1}^{\circ}(x ; v)$ for all $x \in A, U^{\circ}(x ; v)=U_{2}^{\circ}(x ; v)$ for all $x \in B$, and $U^{\circ}(x ; v) \leq \max \left\{U_{1}^{\circ}(x ; v), U_{2}^{\circ}(x ; v)\right\}$ for all $x \in \Gamma$.

\section{PETC MODEL}

We consider the plant model

$$
\dot{x}_{p}=f_{p}\left(x_{p}, u\right),
$$

where $x_{p} \in \mathbb{R}^{n_{p}}$ is the state and $u \in \mathbb{R}^{n_{u}}$ is the control input. We assume that the full state vector $x_{p}$ is measured. Suppose that the following state-feedback controller is designed to stabilize the origin of (3)

$$
\begin{aligned}
\dot{x}_{c} & =f_{c}\left(x_{c}, x_{p}\right) \\
u & =g_{c}\left(x_{c}, x_{p}\right),
\end{aligned}
$$

where $x_{c} \in \mathbb{R}^{n_{c}}$ is the state of the controller. When the controller is static, (4) becomes $u=g_{c}\left(x_{p}\right)$ and there is no need to introduce the state $x_{c}$.

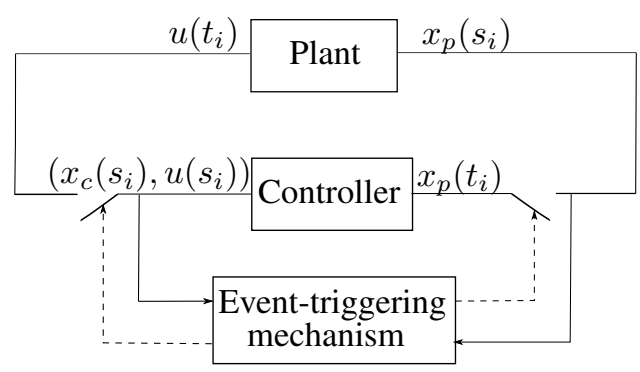

Fig. 1: PETC schematic.

We consider the scenario where plant (3) and controller (4) communicate with each other via a network, see Figure 1. An event-triggering mechanism is used to define the sequence of transmission instants in the following manner. A triggering condition is evaluated at each sampling instant $s_{i}, i \in \mathbb{Z}_{\geq 0}$, where

$$
\varepsilon \leq s_{i+1}-s_{i} \leq T, \quad i \in \mathbb{Z}_{\geq 0},
$$

where $T>0$ is the upper bound on the sampling period and $\varepsilon \in(0, T]$ is the minimum time between two successive evaluations of the triggering condition. When the triggering condition is satisfied, the plant state measurement $x_{p}$ and 
the control input $u$ are respectively sent to the controller and to the plant. Consequently, the sequence of transmission instants, which we denote $\left\{t_{i}\right\}_{i \in \mathcal{I}}, \mathcal{I} \subseteq \mathbb{Z}_{\geq 0}$, is a subsequence of $\left\{s_{i}\right\}_{i \in \mathbb{Z}_{>0}}$, and two successive transmissions are spaced by at least $\varepsilon$ units of time in view of (5), thereby avoiding the Zeno phenomenon. Parameter $\varepsilon$ reflects the minimum achievable transmission interval given by the hardware constraints. We assume that the transmission delays and the quantization effects are negligible. For the sake of generality, we allow the triggering condition to depend on $x_{p}, x_{c}, u$ at the current transmission time. While this may be difficult to implement in practice, this formulation encompasses the practically relevant cases where the controller is directly connected to the actuators and only the plant state is transmitted over the network, or vice versa when the controller is directly connected to the sensors and only the control input is sent over the channel.

Because of the communication network, plant (3) no longer has access to $u$, but only to its networked version, which we denote by $\hat{u}$. Similarly, controller (4) has access to $\hat{x}_{p}$, the networked version of $x_{p}$. Between two successive transmission instants, $\hat{x}_{p}$ and $\hat{u}$ are governed by

$$
\left.\begin{array}{rl}
\dot{\hat{x}}_{p} & =\hat{f}_{p}\left(x_{p}, x_{c}, \hat{x}_{p}, \hat{u}\right) \\
\dot{\hat{u}} & =\hat{f}_{c}\left(x_{p}, x_{c}, \hat{x}_{p}, \hat{u}\right)
\end{array}\right\} \quad t \in\left(t_{i}, t_{i+1}\right),
$$

where $\hat{f}_{p}$ and $\hat{f}_{c}$ are the holding functions. Zero-order-hold devices correspond to $\hat{f}_{p}=0$ and $\hat{f}_{c}=0$ for instance, but other holding functions can be envisioned as well, see [15] for example.

Before modeling the dynamics of $\hat{x}_{p}$ and $\hat{u}$ at each sampling instant $s_{i}$, we introduce the vector $x:=\left(x_{p}, x_{c}\right) \in$ $\mathbb{R}^{n_{x}}$, which is the concatenation of the plant and the controller state, and the vector of network-induced errors $e:=$ $\left(e_{p}, e_{u}\right) \in \mathbb{R}^{n_{e}}$, where $e_{p}:=\hat{x}_{p}-x_{p}$ is the network-induced error on the state measurement $x_{p}, e_{u}:=\hat{u}-u$ is the network-induced error on the control input, $n_{x}:=n_{p}+n_{c}$ and $n_{e}:=n_{p}+n_{u}$. At each sampling instant $s_{i}, i \in \mathbb{Z}_{\geq 0}$, the update of $\hat{x}_{p}$ and $\hat{u}$ satisfy

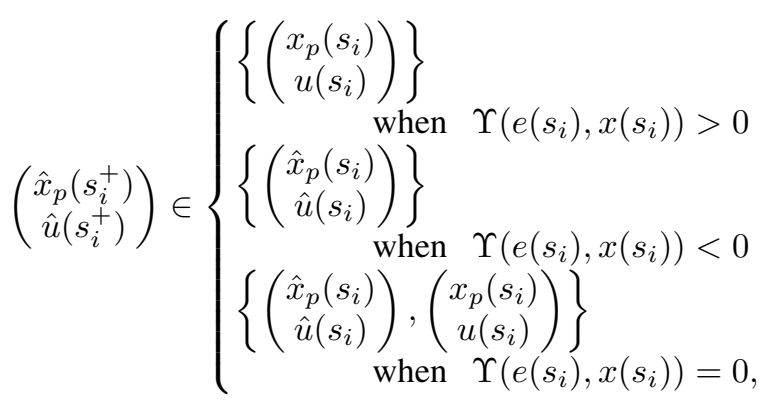

where $\Upsilon$ describes the triggering condition, which is evaluated at each sampling instant by the event-triggering mechanism. We explain later how to construct $\Upsilon$ (see Section IVB). In view of (7), when $\Upsilon\left(e\left(s_{i}\right), x\left(s_{i}\right)\right)>0$, a transmission occurs at $s_{i}$, and $\hat{x}_{p}$ and $\hat{u}$ are reset to the actual value of $x_{p}$ and $u$, respectively. When $\Upsilon\left(e\left(s_{i}\right), x\left(s_{i}\right)\right)<0$, no transmission occurs and $\hat{x}_{p}$ and $\hat{u}$ remain unchanged. When $\Upsilon\left(e\left(s_{i}\right), x\left(s_{i}\right)\right)=0$, the model allows two possibilities: either a transmission occurs or not, our results apply in both cases. This construction ensures that the jump map in (7) is outer semi-continuous, which is essential for the hybrid model presented below to be (nominally) well-posed, see Chapter 6 in [2] for more details. As a result, (7) generates non-unique solutions, which is not an issue for the forthcoming results.

We deduce from (7) that the variable $e$ has the following dynamics at jumps

$$
e\left(s_{i}^{+}\right) \in h\left(e\left(s_{i}\right), x\left(s_{i}\right)\right)
$$

where

$$
h(e, x):=(1-\Gamma(e, x)) e
$$

and $\Gamma: \mathbb{R}^{n_{e}+n_{x}} \rightrightarrows\{0,1\}$ is the function that indicates if a transmission occurs or not. In particular, $\Gamma(e, x)=\{1\}$ when $\Upsilon(e, x)>0$, which corresponds to a transmission. When $\Upsilon(e, x)<0, \Gamma(e, x)=\{0\}$ and this corresponds to no transmission and $h(e, x)=e$ in this case. When $\Upsilon(e, x)=0$, $\Gamma(e, x)=\{0,1\}$ covers the above two possibilities. In agreement with [11], we call (8) the protocol equation. We note that $h$ depends on the state $x$ contrary to [7], [11], [12], which will have important consequences on the stability property of the protocol equation compared to the latter references (see Remark 1 in Section IV-B).

We introduce the variable $\tau \in \mathbb{R}_{\geq 0}$ to keep track of the time elapsed since the last evaluation of the triggering criterium on, which has the following dynamics

$$
\begin{aligned}
& \dot{\tau}=1 \quad \text { when } \quad \tau \in[0, T] \\
& \tau^{+}=0 \text { when } \tau \in[\varepsilon, T] .
\end{aligned}
$$

We then model the complete system as

$$
\begin{array}{cc}
\dot{q}=F(q) & q \in C \\
q^{+} \in G(q) & q \in D,
\end{array}
$$

where $q:=(x, e, \tau)$,

$$
\begin{aligned}
C & :=\mathbb{R}^{n_{x}+n_{e}} \times[0, T] \\
D & :=\mathbb{R}^{n_{x}+n_{e}} \times[\varepsilon, T] .
\end{aligned}
$$

The mapping $F$ in (10) is defined as, for $q \in \mathbb{R}^{n_{x}} \times \mathbb{R}^{n_{e}} \times$ $\mathbb{R}_{\geq 0}$,

$$
F(q):=(f(x, e), g(x, e), 1),
$$

where $f$ and $g$ can be calculated from (3) and (4), and the set-valued mapping $G$ is defined as

$$
G(q):=(x, h(e, x), 0)
$$

with $h$ coming from (9).

Our objective is to design the triggering condition $\Upsilon$ in (7) and to provide an explicit bound on the sampling period $T$ to ensure asymptotic stability properties for system (10).

\section{Main Results}

In this section, we first state the assumption we make on system (10), based on which we then construct the triggering condition $\Upsilon$ and the bound on $T$. We finally present the main stability result. 


\section{A. Assumption}

We assume that system (10) verifies the following properties.

Assumption 1: There exist locally Lipschitz functions $V$ : $\mathbb{R}^{n_{x}} \rightarrow \mathbb{R}_{\geq 0}$ and $W: \mathbb{R}^{n_{e}} \rightarrow \mathbb{R}_{\geq 0}, \underline{\alpha}_{V}, \bar{\alpha}_{V}, \underline{\alpha}_{W}, \bar{\alpha}_{W} \in \mathcal{K}_{\infty}$, $a_{V}, L_{W}>0$ and $L_{V} \geq 0$ such that the following holds.

(i) For all $x \in \mathbb{R}^{n_{x}}, \underline{\alpha}_{V}(|x|) \leq V(x) \leq \bar{\alpha}_{V}(|x|)$.

(ii) For almost all $x \in \mathbb{R}^{n_{x}}$ and all $e \in \mathbb{R}^{n_{e}}$, $\langle\nabla V(x), f(x, e)\rangle \leq-a_{V} V(x)+\gamma^{2} W^{2}(e)$.

(iii) For any $e \in \mathbb{R}^{n_{e}}, \underline{\alpha}_{W}(|e|) \leq W(e) \leq \bar{\alpha}_{W}(|e|)$.

(iv) For any $x \in \mathbb{R}^{n_{x}}$ and almost all $e \in \mathbb{R}^{n_{e}}$, $\langle\nabla W(e), g(x, e)\rangle \leq L_{W} W(e)+L_{V} \sqrt{V(x)}$.

Items (i)-(iii) in Assumption 1 imply that the system $\dot{x}=f(x, e)$ is input-to-state stable (ISS) with respect to $e$. The fact that the Lyapunov function $V$ has an exponential decay rate in item (ii) of Assumption 1 is not restrictive as any input-to-state stable Lyapunov function can be modified accordingly in view of [17]. Item (iii) says that $W$ is positive definite and radially unbounded. Item (iv) is an exponential growth condition of the $e$-system between two consecutive sampling instants, like in [14], [16]. A nonlinear physical example, which satisfies Assumption 1, is provided in Section V.

\section{B. Triggering mechanism}

We define $\Upsilon$ in (7) by, for any $e \in \mathbb{R}^{n_{e}}$ and $x \in \mathbb{R}^{n_{x}}$,

$$
\Upsilon(e, x)=W^{2}(e)-\lambda V(x),
$$

where $\lambda \geq 0$ is a design parameter. We select $\lambda$ such that $\lambda<\lambda^{*}$ with

$$
\lambda^{*}:=\frac{a_{V}}{\gamma^{2}},
$$

where $a_{V}$ and $\gamma>0$ come from Assumption 1 .

Remark 1: The definition of $\Upsilon$ in (14) guarantees that the corresponding protocol (8) is input-to-state stable (see Definition 5.3 in [19]). In particular, $W(e) \geq \sqrt{(\lambda+\nu) V(x)}$ implies $W(h(e, x))=0$ for any $(x, e) \in \mathbb{R}^{n_{x}+n_{e}}$ and $\nu>0$.

For each $\lambda \in\left[0, \lambda^{*}\right)$, we select $T$ in (11) such that $T<$ $T_{\text {MASP }}$, where $T_{\text {MASP }}$ is the maximum allowable sampling period defined as

$T_{\mathrm{MASP}}:=\frac{2}{L_{W}} \operatorname{arctanh}\left(\frac{L_{W}\left(\sqrt{a_{V}}-\gamma \sqrt{\lambda}\right)}{\left(L_{W} \sqrt{\lambda}+2 L_{V}\right) \gamma+L_{W} \sqrt{a_{V}}}\right)$,

where $L_{V} \geq 0, L_{W}, a_{V}, \gamma>0$ come from Assumption 1 . The numerator $L_{W}\left(\sqrt{a_{V}}-\gamma \sqrt{\lambda}\right)$ in (16) is non-negative in view of (15).

The bound in (16) is decreasing in $\lambda$. In other words, the larger the $\lambda$, which leads to a later triggering instant as ensured by (14), the smaller $T_{\text {MASP. }}$. Loosely speaking, this suggest that there is a trade-off between the maximum sampling period and $T$ the number of transmissions generated by the event-triggering condition, typically fast sampling would lead to less frequent transmissions but to more computation and vice-versa.

Remark 2: When $\lambda=0$, the triggering condition $\Upsilon$ is always non-negative, and consequently transmissions can occur at every sampling instants according to (7). Hence, in this case we recover the time-triggered control scenario investigated in [12]. The bound on the MASP in (16) is then

$$
T_{\mathrm{MASP}}=\frac{2}{L_{W}} \operatorname{arctanh}\left(\frac{L_{W} \sqrt{a_{V}}}{2 L_{V} \gamma+L_{W} \sqrt{a_{V}}}\right),
$$

which differs from the one in [12] because the assumptions and the analysis are different. We cannot therefore assess whether one is more conservative than the other in general.

\section{Stability guarantee}

We show that Assumption 1 with a proper selection of $\lambda$ and $T$ ensure the stability of system (10), as formalized in Theorem 1.

Theorem 1: Consider system (10) and suppose the following hold.

1) Assumption 1 is verified.

2) $\lambda<\lambda^{*}$ with $\lambda^{*}$ defined in (15)

3) $T<T_{\text {MASP }}$ with $T_{\text {MASP }}$ defined in (16).

Then, the compact set $\mathcal{A}:=\left\{q \in \mathbb{R}^{n_{x}} \times \mathbb{R}^{n_{e}} \times \mathbb{R}_{\geq 0}: x=\right.$ $0, e=0, \tau \in[0, T]\}$ is UGAS.

Remark 3: The stability property ensured in Theorem 1 is robust to $\rho$-perturbations as the attractor $\mathcal{A}$ is compact and system (10) satisfies the hybrid basic conditions, which implies that it is well-posed, see Chapter 7 in [2].

\section{ILLUSTRATIVE EXAMPLE}

We consider the following van der Pol oscillator

$$
\begin{aligned}
& \dot{x}_{1}=x_{2} \\
& \dot{x}_{2}=\left(1-x_{1}^{2}\right) x_{2}-x_{1}+u,
\end{aligned}
$$

where $x_{1}, x_{2} \in \mathbb{R}$, whose origin is exponentially stabilized by the controller

$$
u=-x_{2}-\left(1-x_{1}^{2}\right) x_{2} .
$$

We consider the case where sensors and actuators are collated via a communication network and the control signal $u$ is submitted to the network and received as $\hat{u}$. Suppose zero-order-hold devices are used to implement the controller, which gives $\hat{f}_{c}=0$ for $\hat{f}_{c}$ in (6). Then, with $e:=\hat{u}-u$ being the networked-induced error (there is no need to introduce $\hat{x}_{p}-x_{p}$ since the controller is static), we obtain the system in (10) with

$$
\left\{\begin{array}{l}
f(x, e):=\left(\begin{array}{c}
x_{2} \\
-x_{1}-x_{2}+e
\end{array}\right) \\
g(x, e):=\left(2-x_{1}^{2}\right)\left(-x_{1}-x_{2}+e\right)-2 x_{1} x_{2}^{2} .
\end{array}\right.
$$

Assumption 1 is satisfied with ${ }^{1} V(x)=3.1783 x_{1}^{6}+$ $3.4385 x_{1} x_{2}^{5}+7.1644 x_{1}^{4} x_{2}^{2}+2.9377 x_{1}^{3} x_{2}^{3}+4.6209 x_{1}^{2} x_{2}^{4}+$ $6.8622 x_{1} x_{2}-0.2499 x_{1} x_{2}^{5}+3.8468 x_{1} x_{2}+1.8511 x_{2}^{6}+$

${ }^{1}$ We have obtained $V, W, \gamma, a_{V}, L_{V}, L_{W}$ using SOSTOOLS [1]. 


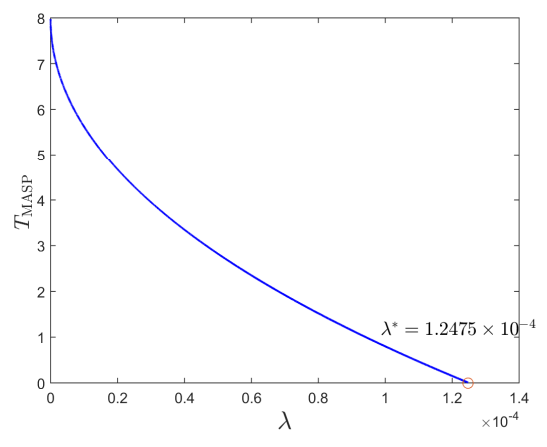

Fig. 2: Triggering parameter $\lambda$ vs $T_{\mathrm{MASP}}$.

$5.3824 x_{2}^{2}, W(e)=|e|, \gamma=8, a_{V}=0.001, L_{W}=L_{V}=$ 0.00071 for $(x, e) \in \mathbb{R}^{3}$. We then calculate $\lambda^{*}$ using (15) which gives $\lambda^{*}=1.2475 \times 10^{-4}$, and for each $\lambda \in\left[0, \lambda^{*}\right)$ we deduce $T_{\mathrm{MASP}}$ from (16). The dependency of $T_{\mathrm{MASP}}$ on $\lambda$ is illustrated in Figure 2.

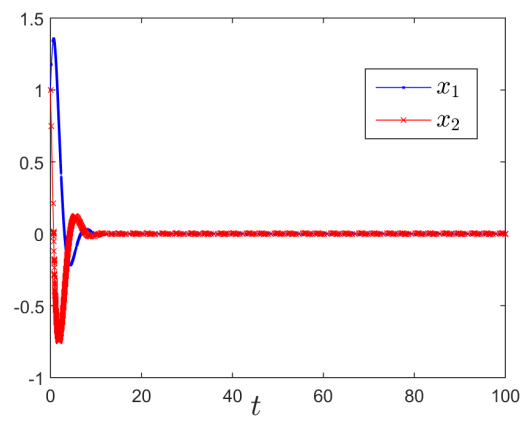

Fig. 3: A solution to system (10) when $T=0.01$ and $\lambda=$ $1.246 \times 10^{-4}$.

We choose $\lambda=1.246 \times 10^{-4}$, which gives $T_{\mathrm{MASP}}=$ 0.0105 and we take $T=0.01$ which satisfies $T<T_{\text {MASP }}$. Figure 3 illustrates the convergence of the plant state to the origin. On the other hand, the convergence no longer occurs when we increase $\lambda$ to $2 \times 10^{-4}$ or when we increase $T$ to 0.1 (which both violate the conditions of Theorem 1). This suggests that the bounds on $T$ and on $\lambda$ are not very conservative for this example.

\section{CONCLUSIONS}

We have addressed the design of periodic event-triggered controllers for a class of nonlinear systems. We have followed an emulation approach for this purpose, in the sense that we start from a given state-feedback controller which stabilizes the origin of the continuous-time plant, and we then explain how to derive a periodic event-triggered condition to preserve stability in the presence of a network. The triggering condition is of the same type as in [20] where continuous event-triggered control was addressed. An easily computable bound on the sampling period used to evaluate the triggering condition is provided. The analysis reveals a trade-off between the parameter of the triggering criterion and the considered sampling period.

\section{REFERENCES}

[1] G. Valmorbida S. Prajna P. Seiler A. Papachristodoulou, J. Anderson and P. A. Parrilo. SOSTOOLS: Sum of squares optimization toolbox for MATLAB. http://arxiv.org/abs/1310.4716, 2013. Available from http://www.eng.ox.ac.uk/control/sostools and http://www.cds.caltech.edu/sostools.

[2] R. Goebel, R.G. Sanfelice, and A.R. Teel. Hybrid dynamical systems: Modeling, Stability, and Robustness. Princeton University Press, 2012.

[3] W.P.M.H. Heemels, M.C.F. Donkers, and A.R. Teel. Periodic eventtriggered control for linear systems. IEEE Transactions on Automatic Control, 58:847-861, 2013.

[4] W.P.M.H. Heemels, G.E. Dullerud, and A.R. Teel. $\mathcal{L}_{2}$-gain analysis for a class of hybrid systems with applications to reset and event-triggered control: A lifting approach. IEEE Transactions on Automatic Control, to appear.

[5] W.P.M.H. Heemels, K.H. Johansson, and P. Tabuada. An introduction to event-triggered and self-triggered control. In Proceedings of the IEEE Conference on Decision and Control, pages 3270-3285, Hawaii, USA, 2012.

[6] W.P.M.H. Heemels, R. Postoyan, M.C.F. Donkers, A.R. Teel, A. Anta, P. Tabuada, and D. Nešić. Periodic event-triggered control. In M. Miskowicz, editor, Event-based control and signal processing, pages 105-119. CRC Press/Taylor \& Francis, 2015.

[7] W.P.M.H. Heemels, A.R. Teel, N. van de Wouw, and D. Nesic. Networked control systems with communication constraints: Tradeoffs between transmission intervals, delays and performance. IEEE Transactions on Automatic Control, 55:1781-1796, 2010.

[8] D. Liberzon, D. Nešić, and A.R. Teel. Lyapunov-based smallgain theorems for hybrid systems. IEEE Transactions on Automatic Control, 59:1395-1410, 2014.

[9] J. Lunze and D. Lehmann. A state-feedback approach to event-based control. Automatica, 46:211-215, 2010.

[10] M. Mazo, A. Anta, and P. Tabuada. An ISS self-triggered implementation of linear controllers. Automatica, 46:1310-1314, 2010.

[11] D. Nešić and A.R. Teel. Input-output stability properties of networked control systems. IEEE Transactions on Automatic Control, 49:16501667, 2004.

[12] D. Nešić, A.R. Teel, and D. Carnevale. Explicit computation of the sampling period in emulation of controllers for nonlinear sampled-data systems. IEEE Transactions on Automatic Control, 54:619-624, 2009.

[13] R. Postoyan, M.C. Bragagnolo, E. Galbrun, J. Daafouz, D. Nešić, and E.B. Castelan. Event-triggered tracking control of unicycle mobile robots. Automatica, 52:302-308, 2015.

[14] R. Postoyan and D. Nešić. A framework for the observer design for networked control systems. IEEE Transactions on Automatic Control, 57:1309-1314, 2012.

[15] R. Postoyan, P. Tabuada, D. Nešić, and A. Anta. A framework for the event-triggered stabilization of nonlinear systems. IEEE Transactions on Automatic Control, 60:982-996, 2015.

[16] R. Postoyan, N. van de Wouw, D. Nešić, and W.P.M.H. Heemels. Tracking control for nonlinear networked control systems. IEEE Transactions on Automatic Control, 59:1539-1554, 2014.

[17] L. Praly and Y. Wang. Stabilization in spite of matched unmodelled dynamics and an equivalent definition of input-to-state stability. Mathematics of Control, Signals, and Systems, 9(1):1-33, 1996.

[18] R.G. Sanfelice and A.R. Teel. Lyapunov analysis of sampled-andhold hybrid feedbacks. In Proceedings of the 45th IEEE Conference on Decision and Control, pages 4879-4884, San Diego, USA, 2006.

[19] M. Tabbara and D. Nešić. Input-output stability with input-to-state stable protocols for quantized and networked control systems. In Proceedings of the 47th IEEE Conference on Decision and Control, pages 2680-2685, Cancun, Maxico, 2008.

[20] P. Tabuada. Event-triggered real-time scheduling of stabilizing control tasks. IEEE Transactions on Automatic Control, 52:1680-1685, 2007.

[21] X. Wang and M.D. Lemmon. Event-triggering in distributed networked control systems. IEEE Transactions on Automatic Control, 56:586601, 2011.

\section{APPENDIX}

Proof of Theorem 1. Let $\lambda \in\left(0, \lambda^{*}\right)$ with $\lambda^{*}$ defined as in (15). Let $T \in\left(0, T_{\mathrm{MASP}}\right)$ and $T_{\mathrm{MASP}}$ be determined by (16). 
We define, for any $q \in C \cup D$,

$$
U(q):=\max \left\{V(x), \phi(\tau) W^{2}(e)\right\},
$$

where $W$ and $V$ come from Assumption 1, and $\phi$ : $\left[0, T_{\mathrm{MASP}}\right] \rightarrow[\underline{\mu}, \bar{\mu}]$ is defined in the next lemma, whose proof is omitted due to space limitations.

Lemma 2: There exist $\bar{\mu}>\underline{\mu}>0$ such that

$$
\underline{\mu}^{*}<\underline{\mu}<\bar{\mu}<\bar{\mu}^{*}
$$

where $\underline{\mu}^{*}:=\frac{\gamma^{2}}{a_{V}}$ and $\bar{\mu}^{*}:=\frac{1}{\lambda}$, and the function $\phi$ defined by the solution to

$$
\dot{\phi}=-2 L_{V} \phi^{\frac{3}{2}}-2\left(L_{W}+\mu\right) \phi, \quad \phi(0)=\bar{\mu}
$$

satisfies $\phi(t) \in[\underline{\mu}, \bar{\mu}]$ for all $t \in[0, T]$.

We first show that the following properties hold for system (10). There exist $\nu>0, \bar{\alpha}_{U}$ and $\underline{\alpha}_{U} \in \mathcal{K}_{\infty}$ such that:

1) $U$ is locally Lipschitz in $x, e$ and $\tau$, and, for all $q \in C \cup D$, $\underline{\alpha}_{U}\left(|q|_{\mathcal{A}}\right) \leq U(q) \leq \bar{\alpha}_{U}\left(|q|_{\mathcal{A}}\right)$

2) for all $q \in C, U^{\circ}(q ; F(q)) \leq-\nu U(q)$;

3) for all $q \in D$ and $\mathrm{g} \in G(q), U(\mathrm{~g}) \leq U(q)$.

It follows from Assumption 1 and the definition of $\phi$ in Lemma 2 that the Lipschitz property of $U$ in item 1) is satisfied. In view of Lemma $2, \phi(\tau) \in[\mu, \bar{\mu}]$ for all $\tau \in$ $[0, T]$. It follows from (21) that item 1) holds with $\bar{\alpha}_{U}, \underline{\alpha}_{U} \in$ $\mathcal{K}_{\infty}$ given by, for all $s \geq 0$,

$$
\begin{aligned}
& \underline{\alpha}_{U}(s)=\min \left\{\underline{\alpha}_{V}\left(\frac{s}{2}\right), \frac{\gamma^{2}}{a_{V}} \underline{\alpha}_{W}^{2}\left(\frac{s}{2}\right)\right\} \\
& \bar{\alpha}_{U}(s)=2 \max \left\{\bar{\alpha}_{V}(s), \frac{1}{\lambda} \bar{\alpha}_{W}^{2}(s)\right\} .
\end{aligned}
$$

We now consider item 2). Let $U_{1}(q):=V(x)$ and $U_{2}(q):=\phi(\tau) W^{2}(e)$ for any $q \in C \cup D$. Let $q \in C$. We distinguish three cases according to Lemma 1.

Case 1: $q \in C$ and $U_{1}(q)>U_{2}(q)$.

In this case, $\phi(\tau) W(e)^{2} \leq V(x)$, hence $W(e)^{2} \leq \frac{1}{\underline{\mu}} V(x)$ according to Lemma 2. Since $\underline{\mu}>\frac{\gamma^{2}}{a_{V}}$ as given in Lemma 2, $\frac{1}{\mu}=\sigma \frac{a_{V}}{\gamma^{2}}$ for some $\sigma \in(\overline{0}, 1)$. As a result, $W^{2}(e) \leq$ $\sigma \frac{a \bar{V}}{\gamma^{2}} V(x)$. Consequently, in view of Lemma 1 and item (ii) of Assumption 1,

$$
\begin{aligned}
U^{\circ} & (q ; F(q))=U_{1}^{\circ}(q ; F(q)) \\
& \leq-a_{V} V(x)+\gamma^{2} W^{2}(e) \\
& \leq-a_{V} V(x)+\sigma \gamma^{2} \frac{a_{V}}{\gamma^{2}} V(x) \\
& =-(1-\sigma) a_{V} V(x)=-(1-\sigma) a_{V} U(q) .
\end{aligned}
$$

Case 2: $q \in C$ and $U_{1}(q)<U_{2}(q)$.

In this case, $U(q)=U_{2}(q)=\phi(\tau) W^{2}(e)$. Hence

$$
\sqrt{V(x)}<\phi^{1 / 2}(\tau) W(e)
$$

We omit below the dependency of $\phi$ on $\tau$ for the sake of convenience. In view of item (iv) in Assumption 1, (22), (25) and the fact that $\phi(\tau) \geq \underline{\mu}$ for all $\tau \in[0, T]$,

$$
\begin{aligned}
U^{\circ}(q ; F(q))= & U_{2}^{\circ}(q ; F(q)) \\
\leq & \left(-2 L_{V} \phi^{3 / 2}-\left(2 L_{W}+\mu\right) \phi\right) W^{2}(e) \\
& +2 \phi W(e)\left(L_{W} W(e)+L_{V} \sqrt{V(x)}\right) \\
\leq & \left(-2 L_{V} \phi^{3 / 2}-\left(2 L_{W}+\mu\right) \phi\right) W^{2}(e) \\
& +2 L_{W} \phi W^{2}(e)+2 L_{V} \phi^{3 / 2} W^{2}(e) \\
= & -\mu \phi W^{2}(e) \leq-\mu \underline{\mu} W^{2}(e) .
\end{aligned}
$$

Since $U(q)=\phi(\tau) W^{2}(e) \leq \bar{\mu} W^{2}(e)$ in this case, (26) gives that

$$
U^{\circ}(q ; F(q)) \leq-\frac{\mu \underline{\mu}}{\bar{\mu}} U(q) .
$$

Case 3: $q \in C$ and $U_{1}(q)=U_{2}(q)$.

In view of Lemma 1, (24) and (27), we have that

$$
\begin{aligned}
U^{\circ}(q ; F(q)) & \leq \max \left\{U_{1}^{\circ}(q ; F(q)), U_{2}^{\circ}(q ; F(q))\right\} \\
& =\max \left\{-(1-\sigma) a_{V},-\frac{\mu \underline{\mu}}{\bar{\mu}}\right\} U(q) .
\end{aligned}
$$

Combining (24) and (27) leads to item 2) with $\nu:=$ $\min \left\{(1-\sigma) a_{V}, \frac{\mu \underline{\mu}}{\bar{\mu}}\right\}$ for all $q \in C$.

We now investigate the evolution of $U$ at jumps, i.e., item 3). Let $q \in D$. We distinguish two cases depending on whether a transmission occurs or not. When a transmission occurs, the corresponding $\mathrm{g} \in G(q)$ is such that $W^{2}(h(e, x))=0$ and thus, since $W$ is positive definite in view of item (iii) of Assumption 1,

$$
\begin{aligned}
U(\mathrm{~g}) & =\max \left\{V(x), \phi(0) W^{2}(h(e, x))\right\} \\
& =V(x) \leq U(q) .
\end{aligned}
$$

When no transmission occurs, it follows from (9) and (14) that $W^{2}(h(e, x))=W^{2}(e) \leq \lambda V(x)$. Since $\phi(0)=\bar{\mu}$ and $\bar{\mu}<1 / \lambda$ according to Lemma 2 ,

$$
\begin{aligned}
U(g) & =\max \left\{V(x), \phi(0) W^{2}(h(e, x))\right\} \\
& \leq \max \{V(x), \bar{\mu} \lambda V(x)\} \\
& \leq \max \left\{V(x), \frac{1}{\lambda} \lambda V(x)\right\}=V(x) \leq U(q)
\end{aligned}
$$

for all $\mathrm{g} \in G(q)$ satisfying $h(e, x)=e$. This with (28) ensures that item 3) holds for all $q \in D$. The satisfaction of items 1)-3) imply that items (i)-(iii) of Theorem 1 in [15] hold and item (iv) of Theorem 1 also holds with noting (11). We then invoke Theorem 1 in [15] and have that system (10) is uniformly globally pre-asymptotically stable (UGpAS).

Note that condition (VC) of Proposition 6.10 in [2] holds for system (10). Moreover, we can exclude item (b) of Proposition 6.10 in [2] in view of items 1)-3), and item (c) of this proposition is also excluded as $G(q) \subset C$ for any $q \in D$ in view of (10)-(13). Then, in view of Proposition 6.10 in [2] and the fact that $t_{j+1}-t_{j} \geq \varepsilon$ holds for any $t, j \in \operatorname{dom} q$, all maximal solutions $q$ of system (10) are complete in $t$ direction, i.e., $\sup _{t} \operatorname{dom} q=\infty$. As a result, the set $\mathcal{A}$ is UGAS for system (10). 